\title{
CAMBIO DE ENERGÍA LIBRE EN LAS REACCIONES BIOQUÍMICAS POR IMPACTO ELECTROMAGNÉTICO
}

Leoncio Molina Vásquez ${ }^{1}$

\section{INTRODUCCIÓN}

El valle de Moquegua abarca parte de la costa alta y zona de quechua, debido al cual tiene un clima cálido. Su temperatura media anual es de $18,7^{\circ} \mathrm{C}$; la media mínima $12,0^{\circ} \mathrm{C}$ y la media máxima anual es de $21,9^{\circ} \mathrm{C}$ (SENHAMI).

La atmósfera del valle presenta muchas partículas de polvo, como se demuestra en la captación de sólidos en suspensión de $45,5 \mathrm{mg} \mathrm{dm}^{-3}$ / 8 horas en la localidad de San Antonio. La velocidad de los vientos varía entre 0 y $7,1 \mathrm{~ms}^{-1}$, siendo por ello suaves y en largos períodos de quietud, especialmente de diciembre a marzo. Presenta una fuerte radiación solar, siendo la humedad relativa promedio anual de $64,8 \%$.

El valle de Moquegua produce árboles frutales, maiz, papa, aji, tomates, alfalfa y otros. También existen molles y restos de flora silvestre.

En este valle se encuentra una línea de trasmisión de energía eléctrica de 10000 voltios que atraviesa cultivos y viviendas. Dentro de estas áreas existen dos cultivos de maíz forrajero experimentales (en la localidad de Homo), uno de maíz tierno y otro, de maíz maduro, atravesados por redes.

\section{ONDAS Y RADIACIÓN ELECTROMAGNÉTICA}

Una línea de transmisión eléctrica se puede definir como un dispositivo para transmitir o guiar energía de un punto a otro. Básicamente tiene dos terminales que alimentan potencia y dos terminales que reciben potencia. Una línea de transmisión es también un dispositivo de cuatro terminales. En una línea de transmisión bifilar, o de dos conductores, los campos eléctrico y magnético no están confinados y se extienden teóricamente hasta el infinito.

\footnotetext{
1. Magister en Química.
}

La energía electromagnética queda establecida por un espectro electromagnético. Las longitudes de onda ionizan el aire y tienen efectos extraños sobre los organismos vivos, porque pueden variar las tasas de crecimiento de células o producir mutaciones en la descendencia de seres vivos.

Las radiaciones electromagnéticas son acumulativas, es decir, la radiación recibida permanece por un largo periodo de tiempo. Cuando se recibe radiación en pequeñas dosis no se siente ningún efecto inmediato, pero a medida que aumenta la cantidad, los efectos se hacen más pronunciados.

\section{PROTECCIÓN BIOLÓGICA ANTE LA RADIACIÓN}

Los métodos de protección son: el blindaje, como sucede en las salas para rayos $X$, donde se construye una pared o mampara forrada de plomo alrededor de los controles de la unidad de rayos $X$ para protección del operador.

El segundo método de defensa es la distancia, debido a que la radiación electromagnética obedece a la ley inversa del cuadrado de la distancia. Por consiguiente, la distancia es un factor extremadamente importante cuando se encuentra una fuente de radiación electromagnética.

\section{LA RADIACIÓN ELECTROMAGNÉTICA Y LAS PLANTAS}

La energia para mantener la vida en la tierra proviene de la luz solar (energía electromagnética), por acción directa en las plantas verdes e indirectamente, a través de otros organismos. La clorofila, gracias a su capacidad de absorber la luz del sol y convertirla en energía química, contenida en las moléculas simples de azúcar, constituye el vínculo de unión esencial entre casi todos los organismos vivientes y la energía electromagnética (solar). 
Esta energía ejerce varios efectos simultáneos en la energía libre $\Delta G$ de las moléculas que producen la diferenciación de los tejidos y órganos. El competidor de esta energía es el agua por su influencia sobre la morfología y anatomía de las plantas.

Toda la energía solar que la tierra recibe está en forma de ondas electromagnéticas, las cuales varian de $290 \mathrm{~nm}$ a $5000 \mathrm{~nm}$.

El espectro visible que atraviesa la atmósfera comprende entre $390 \mathrm{~nm}$ y $750 \mathrm{~nm}$. Con el fin de obtener el máximo de espectro, las plantas han desarrollado pigmentos especiales (clorofila, carotenoides, flavinas, etc.). El fototropismo se rige principalmente por las longitudes de onda azul violeta.

El infrarrojo cercano es importante para las plantas por su influencia en las hormonas que rigen la germinación.

Las radiaciones menores de $390 \mathrm{~nm}$ son demasiado cortas, pero son muy activas en determinadas reacciones químicas.

Las plantas no requieren estas longitudes de onda para su crecimiento $y$, en general, no resultan dañadas por ellas.

La base de comparación es la luz solar para la radiación electromagnética, aunque ésta no es de una intensidad fija, como sí lo son las líneas de transmisión eléctrica de las redes que son la fuente electromagnética de estudio.

En condiciones naturales no se otorga demasiada importancia a las variaciones de longitud de onda, por las siguientes razones:

a) Cada proceso es sensible a las longitudes de onda luminosa, de manera que el análisis de la composición de ondas (amarillas, verdes, azules, etc.) es difícil de interpretar para cada proceso bioquímico.

b) La influencia de la calidad luminosa en las plantas difiere tanto de una especie a otra, no precisando el papel fisiológico de las diferentes partes del espectro.

c) Con las plantas terrestres que crecen en condiciones naturales, no se ha demostrado que las variaciones en la calidad luminosa sean lo suficientemente grandes para ser críticas como factor ambiental.

A pesar de las adaptaciones, por posición, que sufran las partes de la planta, estas sólo necesitan una parte reducida de la energía solar disponible para ser absorbidas en la fotosíntesis.

En cada protoplasto la respiración es un proceso ininterrumpido por medio del cual los compuestos de carbono se oxidan para liberar energía y mantener la actividad vital. Cuando la planta no lleva a cabo la fotosíntesis, su peso seco disminuye progresivamente como resultado de la respiración; la cantidad de luz requerida para la fotosíntesis (ondas electromagnéticas) que puedan igualar el consumo respiratorio de los compuestos de carbono -es decir, para que el $\mathrm{CO}_{2}$ no sea absorbido ni expulsado- se denomina punto de compensación electromagnética (luz).

En el tiempo nublado prolongado, la fotosíntesis puede retardar con respecto a las necesidades de respiración y las reservas alimenticias disminuyen. Es obvio que el crecimiento requiere del exceso de sintesis de la respiración, de tal forma que sus requerimientos mínimos queden satisfechos cuando la intensidad luminosa excede o ha excedido el punto de compensación de luz. Un incremento de luz origina un pequeño aumento en la tasa respiratoria, así como en la fotosintética, e incluso, el efecto neto del aumento de luz, a bajas intensidades, es benéfico, pues la tasa fotosintética aumenta mucho más rápido que la respiratoria.

La energia luminosa inadecuada ejerce una influencia dañina directa cuando los requerimientos respiratorios son mayores.

Cuando las hojas están expuestas a radiación electromagnética intensa (luz solar), los cloroplastos tienden a orientarse de tal forma que sólo un borde está expuesto a la acción directa.

En muchas plantas se ha observado que la intensidad luminosa está directamente correlacionada con la formación de antocianinas, y estos pigmentos que se localizan en las capas superficiales de las células, actúan como pantalla reflectora retardando la penetración de la luz en los tejidos subyacentes. Es decir, las plantas, ante el exceso o deficiencia de luz, tienen compensaciones que les permiten eliminar los riesgos de exceso o deficiencia de radiación solar.

\section{APLICACIÓN A REACCIONES BIOQUÍMICAS CONCERTADAS}

Para la termodinámica, los criterios que se distinguen en un organismo vivo -crecimiento, reproducción, sensibilidad y sostenimiento de una 
estructura altamente ordenada en un ambiente extraño- están relacionados con un gasto constante de energía libre. Esto significa que el organismo (que puede operar isotérmicamente) debe acoplar tareas endergónicas ( $\triangle G$ positivo) a procesos exergónicos ( $\triangle G$ negativo) más amplios.

La célula viva basa la permanencia de su viabilidad en la existencia de ciertos procesos altamente exergónicos (como la respiración o la fotosintesis).

FIGURA № 01: Procesos anabólicos.

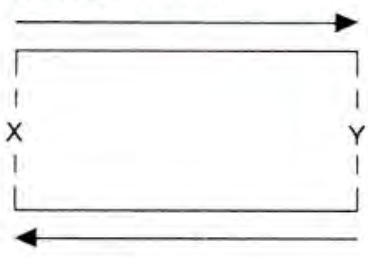

La bioquímica ha identificado el sistema y se ha determinado que es idéntico en todos los seres vivos. La reacción implicada es la sintesis del trifosfato de adenosina (ATP) a partir del difosfato de adenosina (HDP) y fosfato inorgánico $\left(P_{1}\right)$.

$$
A D P+P_{1} \rightleftharpoons A T P \quad \Delta G^{\circ}=+V_{0}
$$

El proceso exergónico "impulsor" es acoplado a esta reacción, la cual procede de modo espontáneo en la dirección de sintesis de ATP; mientras que el proceso endergónico contrario tiene lugar espontáneamente, acoplándolo, también, a la hidrólisis del ATP, para dar lugar a ADP y $P_{1}$. La unidad bioquímica muestra que, independientemente de la fuente de energía aprovechada por los organismos vivos (por ejemplo, los mecanismos de la fotosíntesis de los anaerobios que fermentan alimentos orgánicos), este tipo de reacciones, implican cambios de energía libre. La generación de ATP a partir de ADP y $P$, se efectúa normalmente acoplada a un proceso de oxidación y el mecanismo por el cual un organismo obtiene energía, puede representarse por la ecuación:

$$
\mathrm{AH}_{2}+\mathrm{ADP}+\mathrm{P}_{1} \Longrightarrow \mathrm{A}+\mathrm{ATP}+2 \mathrm{H} \quad \Delta \mathrm{G}^{\circ}=-\mathrm{Vo}_{0}
$$

y los procesos anabólicos por:

$$
\text { Sustrato }(S) \rightleftharpoons \text { Productos }(P) \quad \Delta G^{\circ}=-V_{0}
$$

La función ATP se puede designar:

$$
S+A T P \rightleftharpoons P+A D P+P 2, \Delta G^{\circ}=-V_{0}
$$
diagrama:

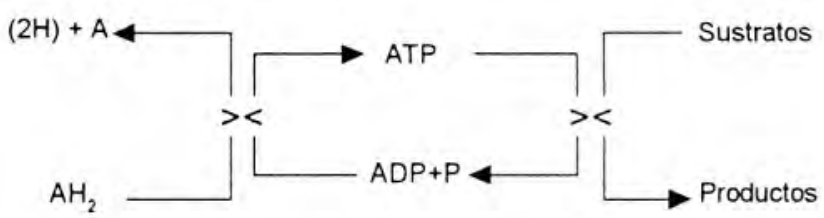

Cuando se usa ATP, por ejemplo, en condiciones estándar modificados $(\mathrm{pH}=7)$, puede efectuarse la sintesis espontánea de glucosa 6-fosfato a partir de glucosa, podría considerarse la reacción como el equivalente de la fosforilación de la glucosa acoplada a la hidrólisis exergónica del ATP.

\begin{aligned} & \hline Endergónicas Glucosa $+P_{1} \rightleftharpoons$ Glucosa 6-fosfato \\ &$\Delta G^{\circ}=+13,8 \mathrm{kJmol}^{-1} \\ &$ Exergónicas $\quad$ ATP $A D P+P_{1} \\ & \triangle G^{\circ}=-31,0 \mathrm{kJmol}^{-1} \\ &$\hline Reacción Neta: Glucosa + ATP $\rightleftharpoons$ Glucosa 6-fosfato + ADP \\ &$\Delta G^{\circ}=-17,2 \mathrm{kJmol}^{-1} \\ &$\hline\end{aligned}

Realmente, no se produce o consume fosfato inorgánico cuando se transfiere un grupo fosfato del ATP a la glucosa en la reacción exergónica $\left(\triangle G^{\circ}=\right.$ $-17,2 \mathrm{kJmol}^{-1}$ ) catalizada por el enzima hexoquinasa.

\section{RELACIÓN ENTRE ENERGIAA Y ENERGÍA LIBRE}

La acción electromagnética genera diferencia de potencial en las plantas. Este cambio de energía $\triangle E$ se relaciona con el cambio de energía libre por:

$$
\triangle G^{\circ}=-n F \triangle E^{\circ}
$$

Además, como $\Delta G^{\circ}=-R T I n K e q$, el valor de $\triangle E^{\circ}$ está relacionada a la magnitud de la constante de equilibrio termodinámico Keq de la reacción de oxidación-reducción.

$$
\begin{aligned}
& -n F \triangle E^{\circ}=-R T I n K e q \\
& \triangle E^{\circ}=\frac{-R T}{n F} \operatorname{lnKeq}
\end{aligned}
$$

Los componentes de los pares redox, en términos de sus concentraciones y no de sus actividades, los trataremos como una celda electromagnetoquímica.

$$
\begin{aligned}
& \Delta G=n F \Delta E_{n} \\
& \triangle E_{n}=E_{n \text { (derecha) }}-E_{n \text { (izquierda) }}
\end{aligned}
$$

Para facilitar la comprensión, $\triangle G$ se considera como la diferencia entre dos términos de energia, uno para cada par redox de la celda $\Delta G_{1 / 2}$ y sus valores se calculan a partir de la ecuación:

$$
\Delta G_{1 / 2}=-n F E_{n}
$$


Donde $\Delta G_{1 / 2}$ puede contribuir por completo a la reacción de la otra media célula; esto es:

$$
\text { oxidante }+ \text { ne } \rightleftharpoons \text { reductor }
$$

para el par redox cuyo potencial es $E_{n}$

También se sabe que el potencial redox, de cualquier par, depende de su grado de oxidación:

$$
\begin{gathered}
\frac{\mathrm{nH}_{2}+\mathrm{ox}}{2} \Longrightarrow \text { red }+\mathrm{nH}^{+} \\
\Delta G_{1 / 2}=\Delta G_{1 / 2}^{0}+\mathrm{RTIn} \frac{[\mathrm{red}]\left(\mathrm{H}^{+}\right)}{[\mathrm{ox}][\mathrm{H} 2] \mathrm{n} / 2}
\end{gathered}
$$

Recordando que $\mathrm{H}_{2}$ y $\mathrm{H}^{+}$son igual a 1, para el electrodo hidrógeno estándar el $\mathrm{pH}$ es cero y el hidrógeno presente a una presión atmosférica estándar es 1.

$$
\Delta G_{1 / 2}=\Delta G^{0}{ }_{1 / 2}+R T \ln \frac{[\text { red }]}{[0 x]}
$$

como $\Delta G_{1 / 2}=-n F E_{n}$

$$
\begin{aligned}
& \Delta G^{0}{ }_{1 / 2}=-n F E^{\circ} \\
& \Delta G^{0}{ }_{1 / 2}=-n F E^{\circ}
\end{aligned}
$$

se tiene $-n F E_{n}=-n F E^{\circ}+R T I n[$ red]

$$
\frac{[\mathrm{red}]}{\text { [ox] }}
$$$$
E_{n}=E^{0}-\frac{R T \ln [\text { red] }}{n F[0 x]}
$$$$
E_{n}=E^{0}+\frac{R T I n \text { [ox] }}{n F \quad \text { [red] }}
$$

usando concentraciones en vez de actividades

$$
E_{n}=E^{\circ}+\frac{R T I n[o x]}{n F \quad[\text { red] }}
$$

Esta ecuación, conocida como la ecuación de Nemst, expresa que si la mayor parte del par redox está presente en forma oxidada, mayor será la tendencia oxidante del par (valor de $E_{n}$ más positivo).

Cuando el oxidante y el reductor de un par redox están presentes en igual concentración, el término In [ox] es igual a cero.

$$
\text { [red] }
$$

Cuando la presión atmosférica es 101,325 $\mathrm{kPa}$ y el $\mathrm{pH}$ es cero, el potencial redox $\left(E^{\circ}\right)$ es por definición $0,00 \mathrm{~V}$; pero si cambia la actividad de los iones hidrógeno $\mathrm{pH} \neq 0$, entonces el nuevo potencial redox tiene el valor $E_{n}$.

$$
\begin{aligned}
& E_{n}=E^{0}+\frac{R \operatorname{Rin}\left(\mathrm{H}^{+}\right)}{n F\left(\mathrm{pH}_{2}\right)} \\
& E_{n}=-0,059 \mathrm{pH}
\end{aligned}
$$

\section{EFECTO DEL En EN EL PH EN CUYA REACCIÓN INTERVIENEN IONES $\mathrm{H}^{+}$}

En muchos procesos redox interviene sólo la transferencia de electrones p.e.

$$
\mathrm{Fe}^{3+}+\mathrm{e} \rightleftharpoons \mathrm{Fe}^{2+}
$$

pero en muchos sistemas biológicos también interviene una transferencia simultánea de iones $\mathrm{H}^{+}$.

$$
\mathrm{A}+2 \mathrm{H}^{+}+2 \mathrm{e} \rightleftharpoons \mathrm{AH}_{2}
$$

Como el $\mathrm{H}^{+}$es un participante en esta reacción, el En de este par debe verse afectado por la actividad de los iones hidrógeno en el medio. De hecho, en este ejemplo, En está relacionado con $E_{0}$ por la siguiente ecuación:

$$
E_{n}=E_{0}-0,059 \mathrm{pH}
$$

En aquellos casos en los que el reductor del par redox es capaz de disociarse como ácido débil como ocurre con muchas sustancias biológicamente importantes- la situación es más compleja porque la disociación está determinada por el pH. Supongamos que el reductor $\mathrm{AH}_{2}$ se disocia como un ácido débil biprótico.

$$
\begin{aligned}
& \mathrm{AH}_{2} \rightleftharpoons \mathrm{H}^{+}+\mathrm{AH}^{-} \\
& \mathrm{AH} \rightleftharpoons \mathrm{H}^{+}+\mathrm{A}^{2-}
\end{aligned}
$$

$\mathrm{AH}_{2}$ permanecerá totalmente sin disociar sólo en medios cuyo $\mathrm{pH}$ es de 6 a 7 y sólo en estos $\mathrm{pH}$ "ácidos" tiene lugar la reacción redox neta.

$$
\mathrm{A}+2 \mathrm{H}^{+} \rightleftharpoons \mathrm{AH}_{2}
$$

Mientras de $\mathrm{pH} 9$ a $10, \mathrm{AH}_{2}$, está completamente disociado en $\mathrm{H}^{+}$y $\mathrm{AH}^{-}$, la reacción neta será:

$$
\mathrm{A}+2 \mathrm{H}^{+}+2 \mathrm{e} \rightleftharpoons \mathrm{AH} \text {. }
$$

a $\mathrm{pH}$ mayores de 12 a $13, \mathrm{AH}^{-}$se disocia completamente y la reacción redox es:

$$
A+2 e \rightleftharpoons A_{2}
$$

Se ha comprobado que cuando intervienen dos protones en la reducción de una sola molécula de oxidante $(\mathrm{pH}<7)$ la curva es lineal.

Para determinar los cambios, se usan indicadores redox. Cualquier materia coloreada (geranio) que se comporte como un par redox reversible puede usarse como un indicador redox, si sus formas oxidada y reducida difieren en color. Los colores deben ser lo suficientemente intensos como para poder usar el colorante en una concentración tan pequeña que su adición a la mezcla de ensayo no cambie significativamente el potencial redox. 
GRÁFICO N 01: Efecto del cambio de pH en el valor de $E_{j}$ a $303 \mathrm{~K}$ para el par redox $\mathrm{AAH}_{1}$ cuando $\mathrm{AH}_{2}$ es un ácido biprótico muy débil, con valores de pK, de 8 y 11.

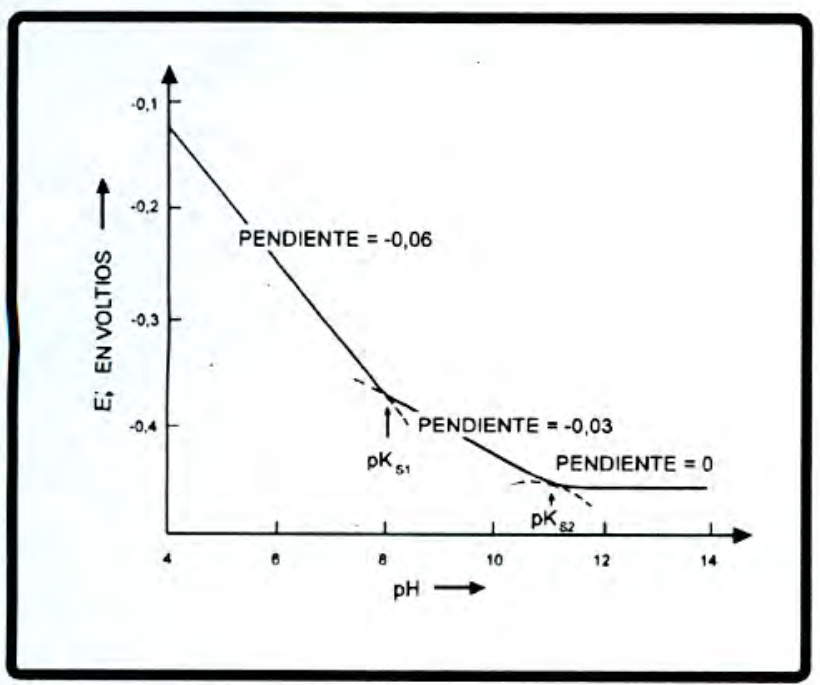

Si el potencial redox En de la planta vegetal a ensayar es En las formas reducida y oxidada del indicador a $298 \mathrm{~K}$, se ajustarán de forma que coexistirán en el equilibrio de la mezcla en las concentraciones dadas por la ecuación.

$$
E_{n}=E_{\text {o(ind) }}+\frac{0,059}{n_{(\text {Ind })}} \log \frac{\text { Ind [ox] }}{\ln \text { [red] }}
$$

Donde Eo es el potencial redox del punto medio del indicador $y$ donde $n_{\text {(ind) }}$ para muchos indicadores redox orgánicos son casi siempre iguales a 2.

Esto significa que un indicador redox no cambia repentinamente de color según se cambia el potencial redox de su medio. El cambio de color es progresivo y se extiende en todo un margen de valores para el potencial redox, el cual se conoce como el "margen de transición" o "intervalo de cambio de color" del indicador.

En un medio cuyo potencial redox es igual a $E o_{(\text {ind) }}$, el indicador presenta color de punto medio (color medio). Si $\mathrm{n}_{(\mathrm{ind})}$ es igual a 2 a $298 \mathrm{~K}$, el indicador presentará potencial redox de $0,06 \mathrm{~V}$ más negativo que el valor de $E o_{(\text {ind) }}$. De forma similar, el indicador presentará un color completamente oxidado en cualquier medio cuyo potencial redox es más de $0,06 \mathrm{~V}$ más positivo que su $E o_{(i n d)}$. El intervalo de $E n$ es de aproximadamente de $0,1 \mathrm{~V}$.

\section{RESPIRACIÓN DE LAS PLANTAS $Y$ ACOPLAMIENTO DE REACCIONES}

En general, la ecuación de la respiración

$$
[\mathrm{HCOH}]+\mathrm{O}_{2}-5 \mathrm{ev} \longrightarrow \mathrm{CO}_{2}+\mathrm{H}_{2} \mathrm{O}
$$

podemos graficarla:

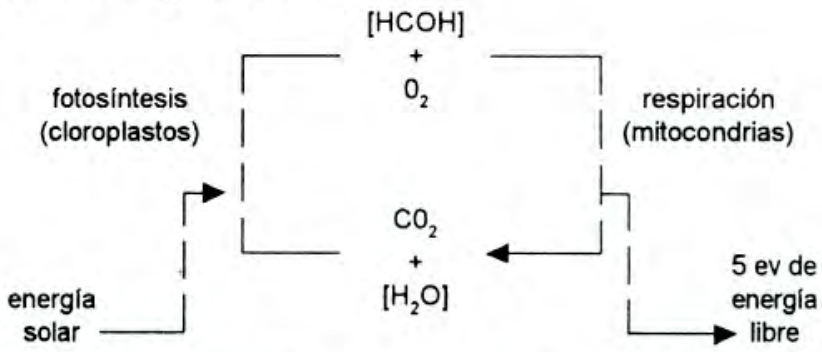

EL CICLO DEL CARBONO COMO ELECTRON VOLTIO

Los procesos dentro de los tejidos vivos implican la formación de moléculas grandes y complejas basadas en otras pequeñas y sencillas. Esta formación de moléculas requiere aporte de energía libre, lo cual significa que las reacciones no son espontáneas. ¿Cómo se suministra energía libre? Acoplando dos reacciones químicas.

La reacción de la glucosa con el oxígeno, para formar $\mathrm{CO}_{2} \mathrm{y} \mathrm{H}_{2} \mathrm{O}$, es espontánea y demasiado lenta; pero la existencia de enzimas apresuran de manera notable estos cambios. La combinación de una molécula de glucosa con seis moléculas de oxígeno libera aproximadamente $30 \mathrm{ev}$. Esta energia libre es suficiente para formar 300 enlaces.

\section{TRABAJO ELÉCTRICO QUE SE LLEVA A CABO EN UNA REACCIÓN ISOTÉRMICA DE OXIDACIÓN}

Toda reacción de naturaleza redox, se compone de:

$$
\begin{array}{ll}
\mathrm{ox}_{1}+\mathrm{ne} \rightleftharpoons \mathrm{red}_{1} & \mathrm{E}_{n}(1) \\
\mathrm{ox}_{2}+\mathrm{ne} \rightleftharpoons \operatorname{red}_{2} & \mathrm{E}_{n}(2)
\end{array}
$$

La reacción espontánea y la f.e.m. positiva de esta célula en su punto potenciométrico nulo es $\Delta E_{n}=E_{n}(2)-E_{n}(1)$.

Se ha subrayado que una célula electroquímica opera en forma termodinámica reversible en su punto potenciométrico nulo. El trabajo producido por una reacción reversible isotérmica y a presión constante, es igual al descenso correlativo en la energía libre de Gibbs del sistema. Cuando se produce una reacción de oxidación -reducción tiene lugar, isotérmicamente, en una célula electroquímica en su punto potenciométrico nulo. El trabajo eléctrico producido por mol de reductor oxidado es igual al descenso de la energía libre de Gibbs por mol, o sea, $-\Delta G=n F \Delta E J^{-1} m^{-1}$ de reductor oxidado. Este ofrece un método para medir el valor de $\Delta G$ para cualquier 
reacción de oxidación-reducción.

En resumen, una reacción de oxidación -reducción isotérmica es:

$$
\text { - } \triangle \mathrm{G}=\mathrm{nF} \triangle \mathrm{E} \mathrm{Jmol}^{-1}
$$

$A E=A E h=f . e \cdot m$. de la reacción oxidación-reducción

\section{TERRENO EXPERIMENTAL}

Los terrenos experimentales se encuentran en la localidad de Homo, valle de Moquegua:

a) El terreno de maiz forrajero tierno tiene una extensión aproximada de $120 \mathrm{~m}$ de largo y $75 \mathrm{~m}$ de ancho; la edad del maiz era de cinco semanas. La línea de transmisión atraviesa dicho terreno y tiene dos postes en dicha área.

b) La extensión del terreno con maiz forrajero a punto de cosechar tiene $83 \mathrm{~m}$ de largo y $53 \mathrm{~m}$ de largo.

\section{MEDICIONES EXPERIMENTALES}

a) Utilizando colorante pulverizado extraido de geranio color fucsia en los tallos de maiz, se nota un cambio de color entre las plantas de maiz sometidas al campo magnético y aquellas plantas de maíz que están fuera de la acción electromagnética.

b) Se realizaron mediciones conductométricas basadas en que la acción electromagnética afecta a las enzimas que modifican la velocidad de reacción en las plantas y alteran el acoplamiento de las reacciones -que producen la energia libre necesaria para la producción de reacciones bioquimicas-. ¿La liberación de 30 ev de energía causa la formación de un enlace químico que

FIGURA N 02: Radiómetro casero.

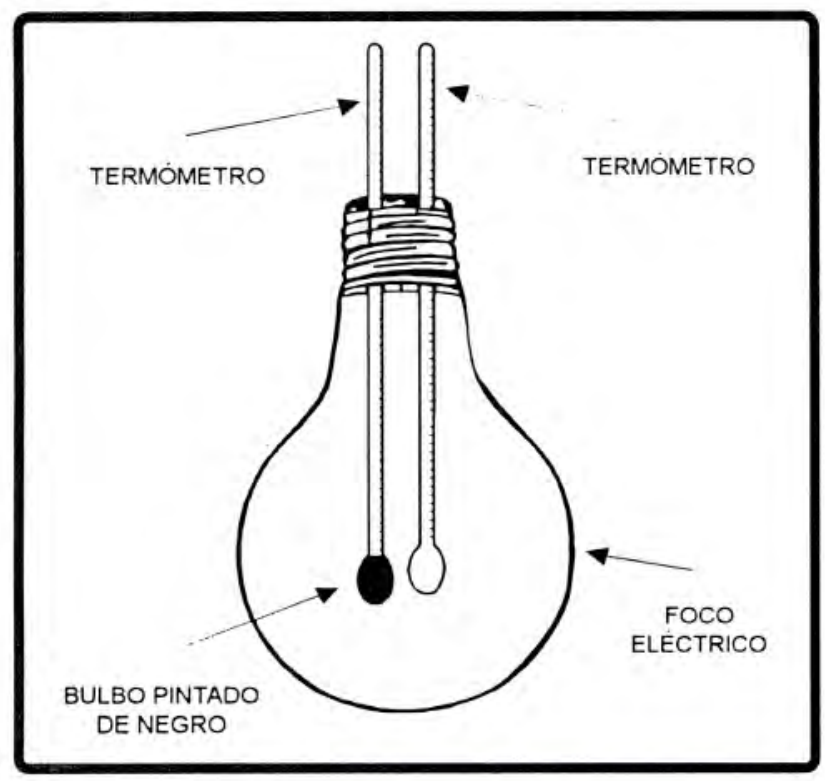

requiere de 0,1 ev? Esto significaria que el 99,7\% de la energia producida por la molécula de glucosa seria desperdiciada. En realidad la glucosa se fracciona lentamente en un proceso de numerosas fases para formar una serie de compuestos. El rango de variación en las diez hileras de maiz maduro fue de 0,03 voltios.

c) Para la experimentación radiométrica, se construyó un radiómetro sencillo colocando dos termómetros en un foco eléctrico modificado, uno de ellos con el bulbo cubierto con pintura negra y otro sin ella.

El instrumento se mantiene en una caja oscura hasta que se lleve a cabo la medición, entonces se saca y se coloca en posición vertical en el área donde se mide la radiación.

El grado de diferencia registrada por los dos termómetros después de cinco minutos puede servir de medida de la energía radiante.

En el caso del maíz forrajero, se encontró variaciones de aproximadamente $0,05 \mathrm{~K}$ en el termómetro expuesto debajo de las líneas de transmisión eléctrica y en lás zonas donde está normalizado el crecimiento de esta planta. No se tuvo termómetros de mayor precisión.

d) En la plantación de maiz tierno en Homo (Moquegua), cuya altura promedio es de $0,80 \mathrm{~m}$ se ha determinado que el crecimiento se ve afectado en las zonas aledañas del poste de transmisión eléctrica, donde muchas plantas son notoriamente más pequeñas y débiles que las plantas de maíz que están fuera o alrededor de las superficies $S_{1}$ y $S_{2}$, por ejemplo,
a) Superficie $S_{1} \longrightarrow$
$\mathrm{N}^{0} 1$
$14,965 \mathrm{~m}^{2}$
b) Superficie $\mathrm{S}_{2} \longrightarrow \mathrm{N}^{\circ} 2$
$17,52 \mathrm{~m}^{2}$

FIGURA $N^{\circ}$ 03: Areas afectadas por postes.

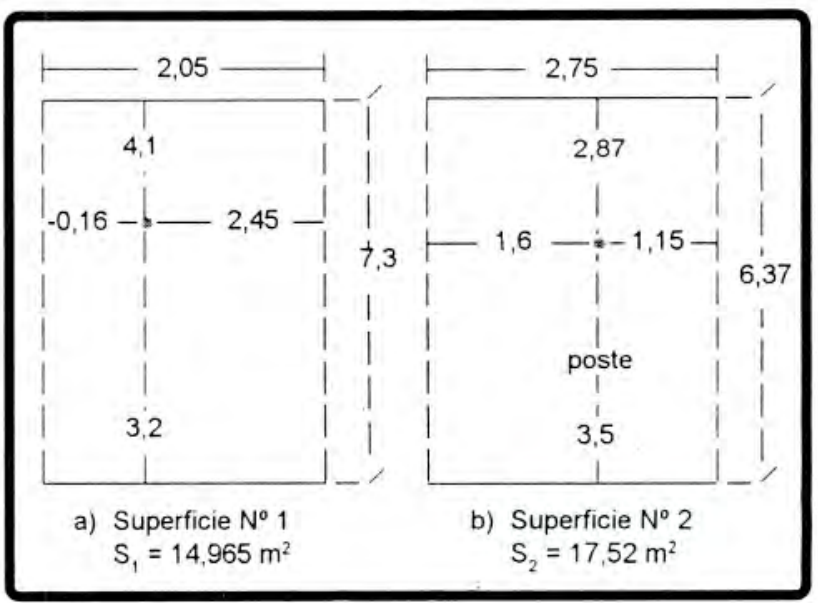


e) También se ha observado en la misma zona de Homo que el crecimiento del maíz maduro se ve afectado, formando una catenaria visible en la superficie verticalmente dependientes de los cables de transmisión como se aprecian en la tabla $\mathrm{N}^{\circ} 1$ y el gráf. $\mathrm{N}^{\circ} 2$.

\section{ANÁLISIS DE LOS RESULTADOS EXPERIMENTALES}

a) La influencia de los campos electromagnéticos de las líneas de transmisión eléctrica sobre los seres vivos (en este caso, las plantas), es notorio cualitativa y cuantitativamente- el diferente grado de acidez en los tallos de maiz; de lo cual se deduce que los equilibrios de las ecuaciones de óxidoreducción se modifican, influenciando en la catálisis enzimática y las reacciones acopladas.

b) La energía electromagnética en las zonas de influencia modifican el espectro solar, a la que se someten normalmente las plantas, al originar una temperatura mayor y traer como consecuencia alteraciones en los mecanismos de acumulación energética en la fotosintesis.

c) El crecimiento del maíz, en las zonas aledañas a los postes y área de influencia electromagnética, lejos de mejorar, como se podría esperar por el incremento en densidad de radiación, disminuye como resultado de la modificación de la energía libre.

d) No se han analizado factores genéticos por su complejidad; pero, es indudable que, al modificarse los mecanismos enzimáticos importantes, la velocidad de las reacciones catalizadas acopladas, producen el desplazamiento de los equilibrios oxired en las plantas y causan influencias aún no conocidas que urgen ser estudiadas.

Teniendo en cuenta que la normatividad en el Código Nacional de Electricidad, no establece precisiones y fundamentos científicos, se deben realizar estudios de impacto electromagnético en las áreas cultivadas por donde se desplazan las lineas

\section{REFERENCIAS BIBLIOGRÁFICAS}

CALDWELL, M.M. y M.L. CALDWELL. A fine Wire Psichrometer for measurement of humidity in the vegetation layer. Ecol 1970.

WASSINK, EC y J.A. STOLWIK. Light quality on plant growth ann rev plant physiol. 1956.

KRAUS, J. Electromagnetismo. Ed. Mc. Graw Hill. México. 1986.

MORRIS, G. Fisico-quimica para las ciencias de la vida. Ed. Reverté. España 1976. de transmisión eléctrica, normando alturas, distancias y tipos de cultivos en las inmediaciones.

TABLA N 01: Modificación de altura del maiz por influencia electromagnética.

\begin{tabular}{|c|c|c|c|c|c|c|c|c|}
$\begin{array}{r}r_{\text {ALIUAA }}^{\text {FILA }} \\
\text { SURCO }\end{array}$ & 1 & 2 & 3 & 4 & 5 & 6 & 7 & 8 \\
\hline 1 & 1,90 & 2,50 & 2,30 & 2,19 & 2,39 & 2,70 & 2,54 & 2,54 \\
2 & 1,96 & 2,74 & 2,66 & 2,60 & 2,32 & 2,73 & 2,60 & 2,70 \\
3 & 2,68 & 2,92 & 2,76 & 2,77 & 2,59 & 2,74 & 2,77 & 2,63 \\
4 & 2,66 & 2,61 & 2,49 & 2,57 & 2,60 & 2,84 & 2,62 & 2,94 \\
5 & 2,58 & 2,78 & 3,00 & 2,43 & 2,74 & 2,95 & 2,92 & 3,38 \\
6 & 2,53 & 3,25 & 2,53 & 2,43 & 2,86 & 2,24 & 2,74 & 3,23 \\
7 & 2,84 & 2,87 & 2,86 & 2,80 & 2,90 & 2,56 & 3,00 & 3,20 \\
8 & 3,04 & 2,85 & 2,38 & 2,65 & 2,76 & 2,76 & 3,03 & 3,23 \\
9 & 2,76 & 2,82 & 2,92 & 2,95 & 2,83 & 2,74 & 2,96 & 3,15 \\
10 & 3,25 & 2,70 & 2,81 & 2,75 & 2,82 & 3,02 & 2,90 & 3,25 \\
PROM. & 2,62 & 2,80 & 2,67 & 2,61 & 2,68 & 3,72 & 2,81 & 3,03 \\
\hline
\end{tabular}

FUENTE: Datos del autor - diciembre - 1993

GRÁFICO N02: Modificación de altura del maiz por influencia electromagnética.

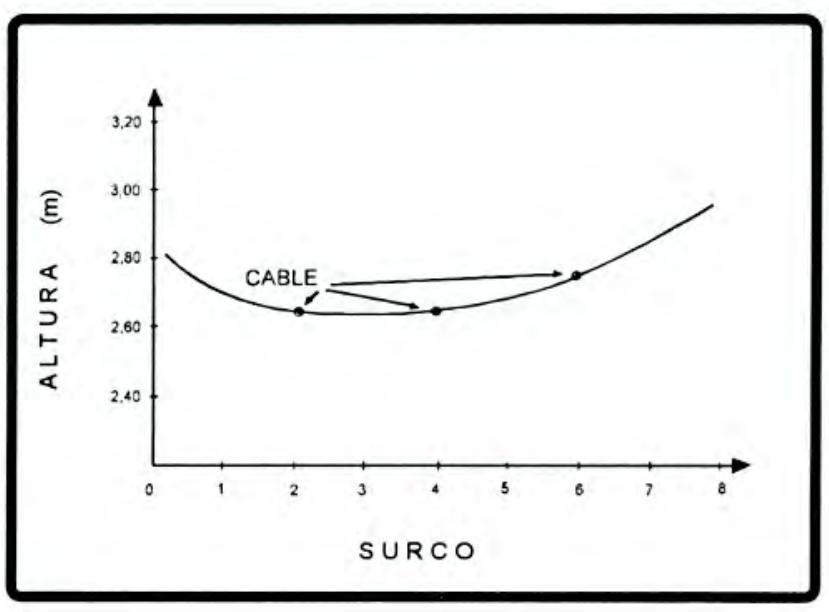

DAUBERNMIRE, R.F. Ecología vegetal. Ed. Limusa. España 1990

OPS. Manual para la localización lógica de fallas y su corrección de equipo para salud. Estados Unidos 1989.

MINISTERIO DE ENERGIA Y MINAS. Código Nacional de Electricidad. Perú 1992. 